Ebisu Ebisu

Études japonaises Études japonaises

$51 \mid 2014$

Le rapprochement franco-japonais dans l'entre-deuxguerres

\title{
Le rôle des juristes japonais dans la fondation de la Maison franco-japonaise
}

日仏会館創立における日本人法律家の役割

The Role of Japanese Legal Scholars in Founding the Maison Franco-Japonaise

Béatrice Jaluzot

(2) OpenEdition

Journals

Édition électronique

URL : http://journals.openedition.org/ebisu/1365

DOI : 10.4000/ebisu.1365

ISSN : 2189-1893

Éditeur :

Institut français de recherche sur le Japon (UMIFRE 19 MAEE-CNRS), Maison franco-japonaise

Référence électronique

Béatrice Jaluzot, «Le rôle des juristes japonais dans la fondation de la Maison franco-japonaise »,

Ebisu [En ligne], 51 | 2014, mis en ligne le 01 novembre 2014, consulté le 30 avril 2019. URL : http:// journals.openedition.org/ebisu/1365; DOI : 10.4000/ebisu.1365

(c) Institut français de recherche sur le Japon à la Maison franco-japonaise 
Le rôle des juristes japonais dans la fondation de la Maison franco-japonaise Béatrice JaLuzot

The Role of Japanese Legal Scholars

in Founding the Maison Franco-Japonaise

Béatrice JALUZOT

\ots-clés : droit, juristes,

Maison franco-japonaise, université, Lyon, relations franco-japonaises, Tomii Masaakira, Suigyama Naojirō.

L'auteure : Béatrice Jaluzot, maître de conférences en droit privé à Sciences Po Lyon, est spécialisée en droit comparé et en droit japonais. Elle vient d'éditer Droit japonais, droit français, quel dialogue? (2014) et poursuit actuellement des recherches sur la diffusion du droit français au Japon.
Résumé : La Maison franco-japonaise qui vient de fêter ses 90 ans est l'une des réussites de la coopération intellectuelle franco-japonaise. Revenant sur ses origines, ce texte rappelle la place importante tenue par les juristes lors de sa fondation. Son initiative ayant été portée par des intellectuels francophiles, cette institution est un témoignage de reconnaissance de la part d'une élite japonaise formée en France, notamment des juristes dont certains ont été formés à Lyon. Nous nous arrêtons ainsi sur Tomii Masaakira, Sugiyama Naojirō ou encore Yamada Saburō afin de rappeler leurs parcours. Ceux-ci contribueront à faire une large place au droit dans les premières activités de la MFJ. 
マキーワード

日仏会館、法律家、富井政章、

杉山直治郎、リヨン市

\section{著者}

リヨン政治学院准教授

1991 年 パンテオン・アッサスパリ第 2 大学

民法学研究科修士課程修了

1992 年 ドイツ、ミュンスター大学 Magister Legum 修了

1993-1996 年 東京大学法学部奨学研究

2000 年 リヨン第 3 大学 法学部博士課程 修了後 法学部准教授に就任

2005 年よりリヨン東アジア研究所研究員

2008 年より現職

\section{要旨}

今年創立 90 周年を迎えた日仏会館は日仏知 的協力事業の成功の一つであろう。本稿では 日仏会館の起源を巡り、その創立時に日本人 法律家が果たした役割を検討する。日仏会館 創設の意図はフランス学問界への謝意表明に あると考えられ、特に法学分野の貢献が大き い。東京大学法学部教授の富井政章や杉山直 治郎といったフランス留学経験者が、留学先 のリヨン大学、リヨン市、フランス人法律家 への感謝の意から日仏会館創設期の活動に関 わった。その結果、会館初期には法学研究が 活発に行われたと考えられる。
\ Keywords: Law, Legal Scholar, Maison Franco-Japonaise, University, Lyon, French-Japanese Relations, Tomii Masaakira, Suigyama Naojirō.

The Author: Béatrice Jaluzot is assistant professor in private law at Sciences Po Lyon. Her field of expertise is comparative and Japanese law. She has recently edited Droit japonais, droit français, quel dialogue? (2014) and her current research topic is the introduction of French law in Japan.

\begin{abstract}
The Maison Franco-Japonaise is celebrating its $90^{\text {th }}$ anniversary this year. It symbolises the success of FrenchJapanese intellectual cooperation. This article aims to remember the role played by legal scholars in the institution's founding through a look back at its roots. The initiative for the MFJ came from Francophile scholars and its creation expresses the gratitude of a Japanese elite educated in France, among them legal scholars trained in Lyon. I will focus on members of this group, such as Tomii Masaakira, Sugiyama Naojirō and Yamada Saburō, and describe their paths. Thanks to these legal scholars from the University of Tokyo, law was an important part of the MFJ's early activities.
\end{abstract}




\title{
Le rôle des juristes japonais dans la fondation de la Maison franco-japonaise
}

\author{
Béatrice JALUZOT*
}

Il est un peu étrange d'aborder la création d'une institution telle que la Maison franco-japonaise sous un angle aussi restreint que celui d'une seule discipline. En effet, s'intéresser à celle-ci uniquement à travers la présence des juristes laisserait à penser que le droit détient une place centrale dans les relations intellectuelles franco-nippones. Une place centrale serait certes inexacte, mais nous pouvons considérer qu'il y occupe un rang important.

Au sein de la Maison et dès sa création, les sciences juridiques sont à l'honneur. C'est justement Bernard Frank qui, à l'occasion des cinquante ans de la Maison franco-japonaise, le rappelle:

« On sait l'importance du rôle qu'avait joué le droit français (...) depuis l'époque de la réouverture du pays et l'influence profonde qu'il avait exercée en dépit du refus de mise en application, par les Chambres, en 1892, du Code Civil préparé par Boissonade. On a pu voir combien, parmi les fondateurs et les animateurs de la Société et de la Maison franco-japonaises, s'étaient montrés actifs des juristes tels que Tomii Masaakira, Sugiyama Naojirō, Yamada Saburō et plus tard, Wakatsuki Reijirō. »(Frank 1974 : 50-51)

Ainsi, les juristes étaient majoritaires au conseil d'administration de l'institution dès sa mise en activité et "c'est... le Droit qui sera durant toute cette période au premier plan des préoccupations de la Maison» (Frank 1974 : 50).

* Sciences-Po Lyon, Institut d'Asie orientale. 
Si les juristes occupent une place un peu à part dans la Maison francojaponaise, il ne faut pas oublier que, symétriquement, ce lieu d'échanges académiques occupe lui aussi une place spécifique, bien que non centrale, dans le monde juridique franco-japonais. Or en France - et peut-être plus quailleurs - les juristes investissent un champ académique qui s'est toujours mis à l'écart, s'éloignant délibérément d'autres disciplines telles que l'histoire, les langues, la littérature ou encore la philosophie. Ceci n'est pas le cas à la MFJ.

Aussi porter l'attention sur les juristes aura certainement l'effet d'une loupe déformante, à la fois sur le rôle que joue la Maison franco-japonaise dans les relations académiques dans ce domaine et sur la place du droit dans l'ensemble de ces échanges. Néanmoins, il nous semble que plusieurs aspects de la Maison franco-japonaise - la place dévolue aux sciences juridiques, leurs liens avec d'autres domaines des sciences sociales, la présence de Lyon en filigrane du projet - peuvent s'expliquer par l'influence qu'ont exercée les juristes japonais lors de la création de la Maison et notre hypothèse est que ces traits sont le reflet de la formation que ceux-ci ont reçue en France et notamment à Lyon.

Il ne s'agit ici nullement de nier la part essentielle qui revient à Shibusawa Eiichi 渋沢栄一 et à Paul Claudel dans cette réalisation : ils ont été les moteurs de cette initiative, l'un grâce à son immense prestige à la fois de financier et de grand philanthrope, l'autre par sa grandeur intellectuelle et son habileté politique ; ce sont eux qui ont mobilisé les volontés politiques et intellectuelles qui ont permis d'aboutir. Néanmoins, l'étude de la place que détiennent les juristes dans l'avènement et dans la réalisation de cette haute institution est justement l'occasion de revenir sur les liens intellectuels forts qui ont été tissés entre Japonais et Français dans le domaine juridique - domaine traditionnellement adjacent au politique - et aussi d'en percevoir les fruits.

Cette étude est également l'occasion d'observer la formidable internationalisation que connaissait le monde d'avant-guerre sous l'angle des relations franco-japonaises. Chacun sait que le degré de mondialisation atteint avant la Première Guerre mondiale était extrêmement avancé, presque équivalent à celui que nous connaissons de nos jours (Marnot 2012). Bien que sa création n'ait eu lieu qu'en 1924, la Maison se présente comme l'un des résultats de cette première mondialisation.

Quelles ont pu être les motivations de part et d'autre ? À dire vrai, le moteur de ces efforts provient certainement de la volonté ardente d'étendre 
le rayonnement à la fois intellectuel, mais aussi politique, des deux pays : de la France en Extrême-Orient et du Japon en France. Le contexte est alors propice à ces échanges, la fin de la Première Guerre mondiale a laissé place à une lutte d'influence dans tous les domaines entre Français et Allemands, chacun cherche à étendre sa sphère dans les domaines académiques (Fillon 2014). Les intellectuels français œuvrent pour un rayonnement international et ce sentiment anime fortement les relations universitaires de l'entredeux-guerres (Audren et Halpérin 2013 ; Durozoi et Bouvet 2009). Par ailleurs, la création de la Maison est aussi le prolongement des relations économiques qu'entretiennent les deux pays. En effet, il semble que l'élévation morale de leur pays ait été une préoccupation des hommes d'affaires d'avant-guerre : ainsi les commerçants lyonnais s'intéressent au Japon, et de même Shibusawa Eiichi mène, parallèlement à sa réussite financière, une œuvre philanthropique.

L'initiative de la MFJ paraît être motivée par un désir d'exprimer une reconnaissance : celle que les francophiles japonais éprouvent vis-à-vis de leurs maîtres français et notamment envers l'Université lyonnaise (I). Cette reconnaissance est plus particulièrement manifestée par les juristes (II).

\section{La Maison franco-japonaise, un témoignage de reconnaissance de la part des intellectuels japonais francophiles}

Un rappel sommaire de l'historique de la fondation est ici nécessaire (Joubin 1929 ; Sugiyama 1936a : 115-119 ; Frank 1974 ; Bouchez 1984 ; Chūjō 2001). Comme son nom l'indique, la Maison est le fruit de volontés convergentes vers un but commun : construire un lieu dédié aux relations intellectuelles franco-japonaises. L'idée initiale vient de la Société francojaponaise de Tokyo qui rassemble au début du $\mathrm{xx}^{\mathrm{e}}$ siècle les intellectuels japonais liés à la France. Elle la soumet à l'ambassadeur de France, Eugène Regnault, qui à son retour en fait la promotion auprès de son gouvernement. Celui-ci manifeste un sentiment favorable au projet en envoyant une délégation universitaire au Japon en 1919. Les représentants de cette délégation, des Lyonnais, vont préciser le projet et le soumettre aux responsables politiques français. Claudel le prend en charge lorsqu'il est nommé ambassadeur au Japon en 1921. 
Parallèlement, à Tokyo, la Société franco-japonaise nomme un comité actif qui entreprend un grand nombre de démarches afin de convaincre les autorités nippones du bien fondé de son initiative. Malgré le terrible séisme qui dévaste Tokyo et sa région en 1923, le comité réunit les fonds nécessaires, tant de la part du gouvernement japonais que de mécènes privés. Des statuts sont soumis aux autorités nippones en janvier 1924 qui consacrent l'existence de cette Maison. L'inauguration officielle a lieu le 24 décembre de la même année en présence d'importantes personnalités japonaises, notamment le prince Kan-in 閑院 et le Premier ministre Katō Takaaki 加藤 高明 (Frank 1974 : 19).

Ce projet est l'initiative d'intellectuels japonais et parmi eux les juristes occupent une place de premier plan ; la proposition trouve un écho favorable auprès de l'Université lyonnaise.

\section{A. Aux origines de la MFJ : le rôle de la Société franco-japonaise de Tokyo, en particulier de Tomii Masaakira}

L'idée de la MFJ est sans nul doute née de la Société franco-japonaise, mais certains auteurs en font aussi revenir le mérite à Tomii Masaakira. Nous tenterons ici de mesurer jusqu'à quel point cette affirmation peut être vérifiée.

La Société franco-japonaise de Tokyo, créée en 1909 (Frank 1974 : 1), rassemble francophiles et anciens élèves de l'école française, alors devenus des personnages influents. En font notamment partie Ume Kenjirō 梅謙次 郎 (1860-1910), Tomii Masaakira 富井政章 (1858-1935), tous deux docteurs en droit de la faculté de Lyon, Yamada Saburō 山田三良 (1869-1965), qui est, avec les deux membres précédents, professeur de droit à l'université impériale de Tokyo, Furuichi Kōi 古市公威 (1854-1934) ${ }^{1}$, ancien élève de l'École centrale de Paris et professeur de cette même université. Le plus influent d'entre eux, Shibusawa Eiichi, avait été, selon Yamada, le premier étudiant envoyé en France par le gouvernement du shogun (Yamada 1929: 36). En 1867, alors trésorier de la mission officielle japonaise, il avait établi des contacts fructueux avec les financiers français et parmi eux, le directeur

1. Il fut le premier directeur du département d'ingénierie de l'université impériale de Tokyo. 
du Crédit lyonnais, dont il rapportera les échanges dans ses souvenirs (Hara 1996 : 113). Il était membre à vie de la Société franco-japonaise depuis le $1^{\text {er }}$ mai 1917 (Shibusawa Eiichi denki shiryō 12 : 261-262).

La Société, soucieuse de diffuser la culture française, suggère alors la " création d'un centre à Tokyo où seraient installés une salle de lecture, des journaux et des périodiques français, une bibliothèque et, peut-être, des conférences" (Frank 1974 : 2). Elle porte cette suggestion à la connaissance de l'ambassadeur de France au Japon depuis 1914, Eugène Regnault (Frank : 1974 ; Joubin 1929 : 948 ; Courant 1919 : 34). Celui-ci apporte son soutien au projet et convainc les autorités françaises. Une délégation universitaire lyonnaise en mission officielle sera ainsi reçue en 1919 chez le vicomte Shibusawa (cf. infra) par plusieurs membres de la Société, notamment les barons Tomii et Furuichi (Shibusawa Eiichi denki shiryō 12 : 266-267).

En mars 1921 la Société franco-japonaise de Tokyo désigne, à la demande de Shibusawa, un comité exécutif qui a la charge de constituer une Maison franco-japonaise (Frank 1974 : 2). Ce comité est largement placé sous l'influence de l'université impériale de Tokyo et de la ville de Lyon. En effet, parmi ses sept membres figurent quatre professeurs de Tōdai : Furuichi Kōi, qui poursuit son soutien au projet, Anesaki Masaharu 姉崎正治 (1873-1949), historien des religions², Sugiyama Naojirō 杉山 直次郎 (1878-1966), qui fait ici son apparition et appartient à la faculté de droit, Émile-Louis Heck (1866-1943), prêtre et professeur de littérature française ${ }^{3}$. Les personnages spécifiquement liés à Lyon sont Kijima Kōzō 木島孝蔵 (il décèdera en 1934), qui vient de quitter ses fonctions de consul du Japon dans cette ville, Tomii Masaakira et Sugimura Yōtaro 杉村陽太郎 (1884-1939) ${ }^{4}$, diplomate, licencié en droit, docteur en sciences politiques de l'université de Lyon depuis 1912.

Tomii semble avoir une place importante dans l'idée initiale d'une telle maison. Selon Sugiyama, il a pesé de toute son influence afin de faire progresser ce projet (Sugiyama et Julliot de la Morandière 1936 : 9). Membre de

2. Il enseignera au Collège de France à la demande de Sylvain Lévi.

3. Heck sera professeur à l'université impériale de Tokyo de 189 à 1921. Il est ordonné prêtre à Tokyo en 1894 .

4. Fillon 2014 : n. 9. Sugimura deviendra vice-secrétaire général à la Société des Nations, directeur des affaires politiques en 1926 puis ambassadeur du Japon en Italie (1934) et en France (1937). 
la Société franco-japonaise depuis sa fondation (Frank 1974 : 2), son nom se retrouve dans toutes les étapes qui aboutiront à la création de la Maison. Avec Shibusawa et Furuichi, il fait partie des représentants du comité qui accompliront plusieurs démarches officielles. Ceux-ci remettent une lettre officielle de demande de subvention au ministère des Affaires étrangères en date du 4 août 1922 (Chūjō et alii 2003 : 190 n. 4) ; le 21 septembre suivant les trois hommes rencontrent le ministre de l'Intérieur Katō et se renseignent sur les conditions d'un espace dédié aux relations franco-japonaises (Shibusawa Eiichi denki shiryō 11 : 261-262). En janvier 1924, les choses s'accélèrent et Tomii fait partie du " comité des fondateurs " (söritsu iinkai 創立委員会) qui compte désormais 18 membres (Frank 1974 : 12). En février suivant, c'est au nom du comité que Tomii, de nouveau aux côtés de Shibusawa et de Furuichi, demande l'autorisation officielle d'implantation de la Maison. Le 7 mars 1924, lorsque le gouvernement japonais attribue la personnalité morale à la Fondation de la Maison franco-japonaise, il reconnaît officiellement ces 18 membres fondateurs (Chūjō 2001 : 24).

L'annonce à Claudel de l'obtention de fonds de la part du gouvernement japonais ainsi que l'obtention de locaux - peu après le terrible tremblement de terre, rappelons-le - est faite par un courrier dont Tomii est là encore le co-signataire (Chūjō 2001 : 24). Dès la première assemblée générale de la Fondation, le 18 mars 1924, Tomii est nommé vice-président du conseil d'administration (Frank 1974 : 15 ; Chūjō 2001 : 24) et Claudel le remercie expressément lors de son discours d'inauguration le 24 décembre (Frank 1974 : 12). Il interviendra à nouveau afin de régler la question du directeur français dont le statut et le rôle restent sujets à mésentente (Frank 1974 : 27). Enfin Tomii devient le quatrième président de la Maison en avril 1934. Il ne reste toutefois à ce poste qu'un an et demi et décède en septembre 1935 (Iyanaga 1984 : 15).

Sugiyama et Julliot de la Morandière, dans l'hommage que la Maison rend à Tomii après son décès, lui attribuent une partie de la réussite de la Maison :

"Lorsqu'après la Grande Guerre, la France envoya au Japon une mission universitaire (...) le Professeur Tomii fut l'un de ceux qui reçurent cette mission, la guidèrent dans ses travaux et lui suggérèrent ses conclusions. On sait que c'est de celles-ci que sortit le premier projet de création à Tokio d'une Maison franco-japonaise, d'un établissement fondé et entretenu par les Japonais où avec la collaboration des deux gouvernements seraient reçus des savants français venant y étudier le Japon et y créer des contacts avec les intellectuels japonais. Le Professeur Tomii fut un de ceux qui 
décidèrent le Vicomte Shibuzawa à s'intéresser à cette fondation. " (Sugiyama et Julliot de la Morandière $1936: 9$ )

Une fois l'œuvre aboutie, c'est aussi Tomii qui souhaite qu'une place importante soit réservée aux disciplines juridiques au sein de la Maison (Sugiyama et Julliot de la Morandière 1936 : 9). Quelques années plus tard, il prendra une part active dans l'accueil d'Henri Capitant au Japon (Ray 1939 : 9).

Quelle part d'influence a pu réellement avoir Tomii Masaakira ? Son parcours porte à croire qu'il a été d'un très grand soutien au projet (Jaluzot 2014). Né en 1858, il avait effectué des études de français à Kyoto sous les enseignements de Léon Dury (Sugiyama et Julliot de la Morandière 1936 : 5). C'est alors qu'il rencontre Émile Guimet, l'industriel lyonnais, qui s'intéresse aux religions nippones et séjourne au Japon en mission officielle entre août 1876 et mars 1877. Tomii lui sert d'interprète (Omoto et Macouin 2001). L'homme d'affaires prend alors Tomii sous sa protection et cette rencontre est probablement à l'origine de la décision du jeune étudiant de venir s'instruire à Lyon. Guimet le soutiendra durant toutes ses études de droit à la toute récente faculté de Lyon, inaugurée en 1875 . Tomii arrive en 1877 et s'avère être un élève remarquable, loué par toute la faculté (Jaluzot 2014). Guimet est à la fois son protecteur et son mécène, car Tomii n'étant pas boursier, il doit subvenir à ses propres besoins. Il lui procure ainsi divers emplois : enseignant de japonais, rapporteur sur le Japon dans différentes sociétés lyonnaises. L'homme d'affaires qui lance alors son projet de musée l'emploie à travailler sur les collections ; Tomii lui rendra d'ailleurs, après son retour au Japon, une étude sur le shintoïsme (1887) (Jaluzot 2014). Ses études sont brillantes et Tomii devient docteur en droit en 1883. Guimet fut un protecteur indispensable à son parcours et l'attachement est réciproque : ses soins sont constants, Tomii est l'invité permanent de la famille et il ne manque pas d'exprimer une vive et touchante reconnaissance :

"Mais, au moins, soyez persuadés, Monsieur et Madame, que vos soins ne seront jamais moralement perdus. Ni le temps, ni la distance, en effet, ne sauraient effacer

5. "Je vous remercie en terminant, de l'extrême bonté que vous avez pour moi en me disant que je puis à tout moment me rendre à Fleurieu : il est probable que je me décide à abuser de votre bienveillante intention " (archives du musée Guimet, lettre de Tomii à Guimet du 26 août 1882). 
le souvenir et la reconnaissance. Et chaque fois que j'aurais à penser à ma jeunesse, à me rendre compte de la cause génératrice de ma carrière, votre nom me reviendra et jamais je ne supporterai les souvenirs aussi touchants sans émotions et sans larmes. " (Jaluzot 2014).

En 1885, deux ans après son retour au Japon, Tomii est nommé professeur à l'université impériale de Tokyo - il a 27 ans - , en droit pénal dans un premier temps, puis en droit civil. Ce premier intérêt pour le pénal explique peut-être qu'il ait gardé des liens avec René Garraud, le pénaliste lyonnais, même près de quarante ans après son départ de Lyon ${ }^{6}$. En parallèle de ses fonctions académiques, il joue aussi un rôle politique en étant nommé à la Chambre des pairs dès 1891, où « il présidera de nombreuses commissions législatives" (Sugiyama et Julliot de la Morandière 1936 : 5). Sa fidélité à ses amitiés françaises ne fait pas obstacle à sa forte volonté d'instaurer un droit japonais moderne et à la pointe de la recherche, ce qui le conduit paradoxalement à jouer un rôle important - voire décisif - dans l'ajournement du Code civil de Boissonade (Okubo 1991 : 399-400).

Ses fonctions deviennent de plus en plus importantes durant l'ère Taishō : selon Sugiyama et Julliot de la Morandière (1936:5), il est nommé à la Cour impériale en 1916, puis juge à la Cour permanente d'arbitrage en 1918 et enfin appelé à siéger au Conseil privé de l'empereur (Sūmitsuin 枢密院). Il est fait baron en 1926, puis promu au jour de sa mort Grand cordon de l'Ordre impérial du Soleil levant.

Nous pouvons ainsi conclure que, sans nul doute, le projet de la MJF est né au sein de la Société franco-japonaise. Mais il est possible que Tomii ait tenu un rôle discret mais important dans sa réalisation. Constituer au Japon un lieu d'échanges culturels et intellectuels, pluridisciplinaire qui permette d'attirer savants et chercheurs français et notamment lyonnais, correspond par beaucoup d'aspects au parcours de Tomii. Son soutien à ce projet peut donc être expliqué par le souhait de saisir l'occasion de marquer, à la fin de sa vie, la gratitude qu'il a envers sa discipline, sa ville d'adoption et l'industriel lyonnais qui lui avait permis de mener à bien ses études à Lyon.

Le fait est que la ville de Lyon est présente très tôt dans l'élaboration du projet de la Maison.

6. Lettre de Tomii à Garraud, 31 août 1920, archives personnelles René Garraud. 


\section{B. Le rôle de l'université lyonnaise}

Le projet de la Maison franco-japonaise est par ailleurs - et en partie le fruit des relations universitaires tissées entre la ville de Lyon et le Japon. Nous avons vu que des Japonais influents, tant universitaires que diplomates, dont une partie a été formée à Lyon, le soutiennent. Du côté de l'université française, le souvenir de ces étudiants reste vif et tout particulièrement ceux qui ont séjourné à Lyon : en 1934, le doyen de la faculté de droit de Paris les évoque, plus de cinquante ans après leur passage sur les bancs de la faculté de droit (Berthélémy 1932 : 494-495). En outre, c’est l'université de Lyon qui est impliquée directement dans cette initiative soutenue par le gouvernement français.

Initialement, l'intérêt du gouvernement de Clemenceau pour la proposition de la Société franco-japonaise est suscité par l'ambassadeur au Japon, Eugène Regnault. Il avait repris cette idée dans un discours prononcé le 5 mars 1914 (Frank 1974 : 3) et à son retour en France en 1918, il propose la création d'une " université française à Tokyo " (Joubin 1929 : 948).

Afin d'étudier la question, le gouvernement décide de missionner une délégation universitaire en Extrême-Orient. Cette mission, ordonnée à la fois par le ministère de l'Instruction publique et celui des Affaires étrangères de Georges Clemenceau, choisit des universitaires lyonnais ${ }^{7}$ qui sont envoyés en Extrême-Orient entre juillet et septembre 1919 (Monestier 1921 : 104). Cette démarche semble assez logique car Lyon entretient alors des relations économiques étroites avec l'Extrême-Orient. La ville qui avait connu son essor grâce à la soie, avait été confrontée à la maladie du ver à soie. Les producteurs lyonnais s'étaient alors tournés vers l'importation et ils avaient noué des liens directs avec la Chine et le Japon dès les années 1860 (Pelletier et alii 2007 : 676). C'est alors l'importation de soie puis de vers à soie du Japon - et aussi de Chine - qui sauvera Lyon de la crise économique (Cœur 2005 : 96). En retour, Lyon deviendra le premier partenaire économique français du Japon jusqu’à la Première Guerre mondiale. La ville est d'une importance telle dans les relations franco-japonaises

7. Bouchez 1984 : 31. L'auteur cite une lettre de Léon Bérard du 30 avril 1921 à son collègue des Affaires étrangères, archives du MAE, série E, cartons 42 et 43. 
qu'un consulat y est installé au début des années $1880^{8}$, avec juridiction sur toute la France (Annuaire Saint-Étienne 1908 : 446).

La délégation française est conduite par le recteur de l'université lyonnaise, Paul Joubin, qui demande à être accompagné de Maurice Courant (Joubin 1929 : 948). Le premier est un ancien élève de l'École normale supérieure en section des sciences, agrégé de sciences physiques et recteur de l'université de Lyon. Le second est professeur à la faculté des lettres de cette même ville et il est l'un des premiers spécialistes de la Corée. Courant avait servi d'interprète autrefois à Tokyo et à Séoul (Lévi 1929 : 415). Le projet de Regnault leur est communiqué (Courant 1919: 34) et leur lettre de mission mentionne le lancement de discussions sur le " rapprochement intellectuel des savants et des universitaires des deux pays, grâce à la fréquentation personnelle et à l'étude réciproque des deux civilisations " (Frank 1974 : 4-5; Chūjō 2001 : 9).

Les universitaires lyonnais trouvent un appui auprès du consulat et ce soutien s'avère décisif. Alors qu’il est à Lyon, le consul Kijima Kōzō (Chūjō 2001 : 14) entretient des relations avec l'université : une lettre adressée à Garraud en date du 29 juillet 1919, en témoigne9. Avant son départ de France, Joubin prend contact avec lui et lorsque Kijima retourne en 1920 au Japon, Joubin le recommande auprès de la Société franco-japonaise (Sugiyama 1936b : 116). Kijima prend dès lors une part très active dans l'aboutissement du projet. Il deviendra la cheville ouvrière de la Maison et son administrateur gérant jusqu’à sa mort en 1934 (Frank 1974 : 4). Ceux qui ont côtoyé Kijima en gardent un souvenir fort, celui de l'âme de la Maison. Sylvain Lévi, en 1929, l'évoque en ces termes : «l'année suivante, le Consul du Japon à Lyon, M. Kijima, rentrait en congé ; nul n'était mieux doué pour faire aboutir le projet. Il se mit à l'œuvre avec passion, il sollicita avec une infatigable obstination les concours nécessaires " (Lévi 1929 : 415).

Alors qu'ils sont à Tokyo, Joubin et Courant sont introduits dans des cercles très influents et ils rencontrent un grand nombre de dirigeants tant

8. Yamada "Tadzumi » est le premier consul du Japon à Lyon, il avait épousé une lyonnaise, Marguerite Varot et de cette union est née la poétesse Kikou Yamata. Il décède dès son retour au Japon en 1917 (Reyns-Chikuma 2005 : 76 ; papiers Kikou Yamata).

9. Nous remercions Catherine Fillon de nous avoir transmis cette archive. 
politiques qu'universitaires, dont le Premier ministre Hara Takashi 原敬 (Chūjō 2001 : 9, citant les archives diplomatiques de Kijima). Shibusawa Eiichi organise en leur honneur un dîner (Yamada 1929 : 36) à la résidence Hatoyama, le 4 août 1919 en présence de personnalités influentes et " connues pour leurs sympathies françaises" (Lévi 1929 : 415) : Hozumi Nobushige 穂積陳重 ${ }^{10}$ et Tomii Masaakira, rédacteurs du Code civil japonais, professeurs de droit à l'université impériale de Tokyo, Inukai Tsuyoshi 犬養毅, chef du parti national constitutionnaliste ${ }^{11}$ (Rikken kokumin tō 立憲国民党), Sakatani Yoshirō 坂谷芳郎, ancien ministre des Finances, sénateur et gendre de Shibusawa, et Furuichi Kōi, ancien élève de l'École centrale de Paris, professeur à l'université impériale de Tokyo et président de la Société franco-japonaise (Shibusawa Eiichi denki shiryō 12 : 266-267).

À l'occasion de ce dîner, Joubin suggère le principe d'une institution à mettre en œuvre de manière commune (Shibusawa Eiichi denki shiryō 12 : 266-267). Ils rendent une note à Shibusawa le 22 septembre 1919 dans laquelle ils disent souhaiter développer des " moyens de pénétration intellectuelle réciproque " et évoquent plusieurs objectifs dont le but d'ensemble est l'attraction de chercheurs français au Japon. Les interlocuteurs japonais ont visiblement accordé l'assurance d'un accueil de très grande qualité à ceux-ci ${ }^{12}$. Sur cette base, Joubin dresse un projet qu'il soumet au gouvernement et il obtient la promesse de 300000 francs (Joubin 1929 : 949). L'annonce en est faite au ministère des Affaires étrangères le 2 juin 1921 (Frank 1974 : 5-8). Claudel, alors qu'il se prépare à prendre son poste, est

10. Il est le gendre de Shibusawa dont il avait épousé la fille aînée ; nous remercions le professeur Ōmura Atsushi de l'université de Tokyo de nous avoir transmis cette information.

11. Qui deviendra Premier ministre et sera assassiné par les nationalistes.

12. "La deuxième (condition), c'est que ces envoyés français soient assurés d'avoir toutes facilités pour remplir leur mission d'études et d'échanges intellectuels, c'est-àdire qu'ils trouvent des sympathies actives qui les guident amicalement, leur ouvrent les portes nécessaires, les introduisent dans la société japonaise, les mette en relation avec les savants, les professeurs, les ingénieurs etc., en un mot des hôtes, qui soient à la fois leur soutien moral et leurs garants devant l'opinion publique ", " nous sommes certains, après les visites que nous avons faites aux plus hautes personnalités politiques, aussi bien qu'aux savants et aux universitaires de ce pays, que ce patronage ne leur ferait pas défaut "; note pour M. le baron Shibusawa, remise à la date du 22 septembre 1919, archives du Bureau japonais de la MFJ, citée par Frank (1974 : 5-6). 
vivement intéressé par le projet et il rencontre Joubin ${ }^{13}$ auquel il en attribue la paternité : "Monsieur Joubin, (...) est en somme, l'auteur du projet » écrit-il le 8 novembre 1922 (Chūjō et alii 2003 : 192). Les deux hommes restent en contact durant l'élaboration du projet ${ }^{14}$, bien que Joubin se soit mis en retrait et ait préféré se consacrer aux relations franco-chinoises (Courant 1919).

Le tissage de ces relations explique ces liens spécifiques de la Maison franco-japonaise avec la ville de Lyon dont l'ombre règne sur l'institution dès la pose de ses fondations : il y est notamment prévu que le quatrième pensionnaire de la future Maison soit demandé à la chambre de commerce de Lyon $^{15}$.

Mais ce n'est qu'en 1924 que le projet sera concrétisé, cette réalisation devant beaucoup aux juristes.

\section{La Maison franco-japonaise, un acte de reconnaissance de la part des juristes japonais}

Cette reconnaissance se manifeste par l'élaboration des statuts de la MFJ par un professeur de droit, Sugiyama, d'une part et par l'activité scientifique de la Maison d'autre part.

A. La structure originelle de la Maison franco-japonaise : une œuvre des juristes japonais, en particulier de Sugiyama

Les fondateurs japonais apportent un soin tout particulier à la rédaction des statuts de la Maison ; l'institution permet d'instaurer des relations mutuelles entre les deux pays, et dans le domaine juridique elle devient un carrefour d'échanges qui contribue à faire connaître le droit français au Japon et le droit japonais en France.

13. AMAE E/63/divers 1-2, cité par Chūjō et alii $2003: 188$.

14. Chūjō et alii 2003 : 188. L'auteur cite une lettre du 16 janvier 1922 par laquelle Joubin propose à Claudel de nommer Henri Focillon à la tête de la MFJ.

15. Archives de la MFJ : texte des résolutions adoptées par le comité japonais de la Maison franco-japonaise, le 29 juillet 1925. 
Ainsi que nous l'avons vu, Sugiyama attribue les mérites de la création de la Maison à Tomii ; il joue pourtant lui-même un rôle essentiel dans le succès du projet, en tant que membre actif du comité exécutif chargé du projet, et prend une part active dans la rédaction des statuts de la Maison (Chūjō et alii 2003 : 190, n. 7). Il est très influent, à la fois dans les relations avec les partenaires français et dans les propositions concrètes qu'il élabore et présente aux autorités japonaises.

C'est notamment lui qui est à l'origine de l'insertion de la réciprocité au sein même de la Maison. Au départ les Français n'avaient pas une telle conception, le modèle initial était plutôt celui des écoles françaises de Rome ou d'Athènes ${ }^{16}$. Le projet de Joubin était en conséquence celui d'une "Maison de France ", ce que Claudel reprend à son compte. Joubin avait même proposé au gouvernement japonais des statuts provisoires en ce sens. La réciprocité était prévue, mais par la création d'une autre Maison à Paris : la "Maison du Japon à Paris " (Joubin 1929 : 948). Du côté japonais, selon les propos de Sugiyama, le projet initial était celui d'un établissement fondé et entretenu par les Japonais, qui recevraient des savants français afin qu'ils étudient le Japon et puissent y créer des contacts avec les intellectuels japonais (Sugiyama et Julliot de la Morandière 1936 : 9). Cependant la réciprocité devait être présente $a b$ initio à Tokyo et le nom proposé en conséquence était celui de " Maison franco-japonaise ». Sugiyama convainc Claudel et le conduit à accepter cette appellation (Chūjō et alii 2003 : 183). Le célèbre poète écrit ainsi le 2 novembre 1922 à Eugène Aurousseau :

"M. Sugiyama, dans le projet qu'il a remis au Ministre de l'Instruction Publique, a notablement élargi mon programme. Il a prévu que les personnes en résidence à l'Institut pourraient être

$1^{\circ}$ des savants français désignés par le Gouvernement Français (avec leur famille); $2^{\circ}$ des jeunes gens français venus au Japon pour y faire un stage d'étude et d'enseignement ;

$3^{\circ}$ les Français venus au Japon dans un but d'études et qui voudraient y séjourner plus d'un mois ;

$4^{\circ}$ des étudiants japonais dans la limite d'un séjour d'au moins 6 mois.

Cela me parât acceptable. » (Chūjō et alii 2003 : 190).

16. Note remise par P. Joubin et M. Courant au vicomte Shibusawa, citée par B. Frank (1974: 5) : "Ce serait une sorte d'école analogue à ces écoles de Rome ou d'Athènes, qui ont tant fait pour la connaissance des civilisations occidentales. » 
Un "Plan officieux sur le projet de la Maison franco-japonaise " (Nichifutsu kaikan mokuromi shian 日仏会館目論見私案 ${ }^{17}$ ) est déposé le 25 octobre 1922, Sugiyama est l'auteur de l'avant-propos. Mais ce n'est qu'un an après, en janvier 1924, que l'Association pour la fondation de la Maison franco-japonaise rédige des statuts. Le 7 mars de la même année, la Maison obtient la personnalité morale et le gouvernement prend acte des 18 membres fondateurs. Lors de la première assemblée générale, le 18 mars suivant, 20 conseillers et 20 administrateurs sont nommés, Shibusawa en devient le président (Chūjō 2001 : 23-24) et Sugiyama, l'administrateur (Frank 1974 : 16).

Une certaine asymétrie dans ces relations fondatrices est perceptible. Les juristes japonais, éduqués à l'école française, ont porté une attention spécifique à la structure juridique de la Maison. Celle-ci est parfaitement structurée du côté japonais, ce qui s'explique aussi par le fait que la MFJ est une personne juridique de droit japonais. Du côté français, il existe un net déficit - explicable encore par le fait que la MFJ n'est pas une personne juridique française - qui sera à l'origine de bien des tensions (Vincent 1995 : 6-16). Néanmoins, le besoin de clarifier les règles ne semble pas avoir réellement préoccupé les intellectuels français chargés de la direction de la Maison. En 1974, Bernard Frank exprime sa perplexité à cet égard car les juristes français qui se sont succédés à la tête de la Maison ont paru se satisfaire d'une situation qui semble pourtant être source d'insatisfaction :

"Paradoxalement, loin de se sentir gênés par le fait que n’ont jamais été codifiées les règles qui régissent dans la Maison les rapports des deux parties et qui "résultent, pour la plus grande part, de conventions et d'usages peu à peu établis entre les Japonais et les Français qui y cohabitent", les directeurs successifs et, précisément, les juristes, qui sont les mieux placés pour donner une opinion en la matière, se sont, après un certain temps d'expérience, déclarés profondément satisfaits de "la souplesse inégalable de son organisation première", "de cette organisation coutumière (qui) répond à la logique de l'institution et en assure finalement le bon fonctionnement" [René Capitant] (...)

À quoi tient [la] réussite [de la Maison franco-japonaise] ?, essentiellement à l'absence de toute constitution écrite précisant les modalités exactes de la collaboration franco-japonaise, à la souplesse de son fonctionnement, à la non-codification de ses règles. » (Frank 1974 : 30).

17. JACAR, numéro de référence B03041009100, 外務省外交史料館, 在内外協会関係雑 件／在内/部第一巻 (B-1-3-3-005). 
Ainsi que nous venons de le voir, Sugiyama Naojirō a pris une part très importante dans la création de la Maison. Quelles ont pu être les motivations qui l'ont conduit à déployer une telle énergie pour ce projet ? Sugiyama naît à Tokyo le 29 janvier 1878 et décède le 15 février 1966. Il étudie le droit à l'université impériale de Tokyo dont il est licencié en 1903 et où il devient l'élève de Tomii. Avant d'être envoyé à l'étranger, il enseigne le droit romain et le droit français dans diverses universités. Son séjour en Occident se répartit dans plusieurs pays : en Allemagne, en Suisse, aux États-Unis et en France, où il effectue deux séjours, en 1905 et en 1925. Selon Julliot de la Morandière, il est alors séduit par " la hauteur de vue et la clarté des juristes français " (Julliot de la Morandière 1966 : 925) et devient dès lors un fervent artisan des relations entre universitaires français et japonais. Le juriste français affirme que c'est au cours d'un passage à Paris que Sugiyama entend les leçons d'Henri Capitant et qu'il s'arrête plusieurs mois pour les écouter :

"Celui-ci, venu en Europe avant la guerre, avait passé la presque totalité de son séjour à Berlin. Avant de se rembarquer, selon la recommandation de son Maître, il vient à Paris et suit quelques cours à la Faculté de Droit. Il entend Capitant. Et c'est pour lui l'illumination. Il est conquis par la clarté des raisonnements, l'élévation des idées générales... Son but dans la vie sera désormais le rapprochement entre la culture orientale et la culture occidentale par l'intermédiaire du Droit français. " (Julliot de la Morandière 1939 : 21)

En août 1915, il est nommé professeur à la faculté de droit de l'université impériale et, dès septembre 1916, il succède à son maître (Julliot de la Morandière 1966 : 923). Certains de ses élèves suivront son chemin : Ishizaki Masaichirō 石崎政一郎 ${ }^{18}$ sera l'auteur en 1927 d'une thèse pionnière en droit du commerce international à Lyon sous la direction d'Edouard Lambert (Osman 2014).

Sugiyama devient vice-président du Conseil japonais de la Maison en 1928, puis administrateur général de la direction scientifique en 1930 (Julliot de la Morandière 1966 : 925), avant de prendre sa retraite en avril 1938. Il a été décoré de la Croix d'officier de la Légion d'honneur en 1923 ,

18. Cf. lettre d'Ishizaki à Louis Josserand, 4 juillet 1937, remise par Catherine Fillon : "mon maître Sugiyama..." ". 
puis la Cravate de commandeur. Sugiyama verra ses mérites hautement récompensés par l'Université française. Il devient docteur honoris causa de l'université de Paris en 1932 (Allix 1934 : 186) et de Lyon en 1933 (Annuaire de l'université de Lyon 1934 : 23), puis de Bordeaux en 1934 (Malherbe 1996 : 232).

Mais son action ne se limite pas à la seule fondation de la Maison, pendant longtemps, il en anime l'activité scientifique et il y laisse une large part au droit.

\section{B. L'activité initiale de la MFJ : une large place faite aux juristes}

La création de la Maison permet de promouvoir des liens forts entre juristes japonais et juristes français, ce qui conduit à une importante présence de ces derniers. En naîtront des amitiés fortes, réciproques et fructueuses, qui donneront lieu à de riches échanges intellectuels qui perdureront au-delà de la Seconde Guerre mondiale.

Sugiyama demande que « les liens avec le droit français soient renoués». Il invite notamment Henri Capitant dont la mission au Japon sera l'un des événements marquants de la jeune Maison. Les communications du célèbre juriste sont publiées en japonais en 1931 dans la revue Nichifutsu bunka ( $\mathrm{n}^{\circ} 1$ Nouvelle Série), en particulier «Les transformations du droit civil français depuis cinquante ans" (Capitant 1931).

Ces liens vont toutefois être durement éprouvés durant la guerre. L'institution devra faire face à l'avènement du nationalisme et à la détérioration des relations entre nos deux nations. Nous en trouvons des traces dans les archives de la Maison : en 1937, Léon Mazeaud envoie une lettre au ministère des Affaires étrangères afin de soutenir le maintien de Georges Bonneau, docteur en lettres japonaises de l'université impériale et collaborateur de la Maison, qui souhaite rester au Japon jusqu'en 1940, comme il était prévu. Le gouvernement souhaitait interrompre la mission (archives de la MFJ, lettre du 16 novembre 1937). L'institution reste fidèle à ses engagements et subit les affres de la guerre (Joüon des Longrais 1947 : 294, 300-301). Sugiyama est conscient de ce qui se passe :

"Avec le baron Tomii (...), il voyait avec inquiétude se développer au Japon un mouvement tendant vers un nationalisme outrancier. Tout en reconnaissant aux militaires japonais d'immenses qualités de désintéressement personnel, de dévouement 
absolu à la Patrie, il redoutait leur aveuglement. "Ils vont tout compromettre" (...) sa fidélité à la France ne se démentit jamais. Au cours de la dernière guerre, il fut suspect au Gouvernement du Japon (...) mais au cours des interrogatoires que lui firent subir la police, et l'on devine ce que je veux dire, il refusa de renier ses amitiés françaises et il ne cessa de protéger du mieux possible les Français qu'il connaissait. " (Julliot de la Morandière 1966 : 926).

La direction française de la Maison est occupée à plusieurs reprises par des universitaires français issus des facultés de droit. Peu de temps après l'avènement de la Maison, son conseil d'administration demande à ce que le directeur français soit un juriste, ainsi quand il établit, en mai 1929, une liste de personnalités susceptibles d'être nommées à ce poste par le comité français de la Maison, elles le sont toutes, sans exception ${ }^{19}$. Pourtant aucun d'entre eux ne le devient : Louis Josserand avait accepté le poste, puis il est revenu sur sa décision, invoquant une santé fragile (archives du Rhône 224 J 1-67), et c'est Joseph Hackin (1886-1941), archéologue et conservateur du musée Guimet depuis $1923^{20}$, qui est nommé directeur français. Toutefois les directeurs suivants seront très souvent professeurs de droit : Léon Julliot de la Morandière (1933-1936), Léon Mazeaud (1936-1939), Frédéric Joüon des Longrais (1939-1946), René Capitant (1957-1960), Jacques Robert (1966-1968), et enfin Michel Lesage (1974-1977).

Après guerre, François Chabas, alors doctorant en droit, en devient pensionnaire (Julliot de la Morandière 1966 : 925) et sa thèse, soutenue en 1933, porte sur le droit comparé franco-japonais de la responsabilité civile.

En raison des grandes qualités de communication que manifestait Sugiyama - ses discours en France en témoignent - on aurait pu craindre que sa retraite ait un impact négatif sur la présence des juristes au sein de la Maison, or le relais est assuré par Yamada Saburō. Présent aux origines de la

19. Archives de la Maison franco-japonaise, "mémoire ", Tokio, le 3 mai 1929 : les noms proposés sont : Ferdinand Larnaude, doyen honoraire de la faculté de droit de Paris, Henri Capitant, François Gény, René Demogue, Georges Ripert, professeurs à Paris, Edouard Lambert, Louis Josserand et Paul Pic, professeurs à la faculté de Lyon, Julien Bonnecase, alors Doyen de la faculté de Bordeaux (Malherbe 1996 : 156.), Charles Gide, économiste alors au Collège de France.

20. Il décèdera en 1941 avec son épouse, elle aussi archéologue, sur un navire torpillé par la marine allemande au large de la Bretagne. http://www.chine-informations.com/ guide/joseph-hackin_3191.html 
Maison, il paraît n'y avoir occupé aucune fonction durant sa carrière académique, mais il revient ensuite pour en devenir l'un des présidents japonais les plus logtemps en poste. Là encore nous rencontrons un parcours hors norme. Yamada était lui aussi professeur de droit à l'université impériale, spécialiste de droit international privé, domaine particulièrement stratégique au Japon. À plusieurs reprises, il prend part à des événements décisifs pour l'avènement du Japon sur la scène juridique internationale. Ayant été envoyé en Europe afin d'y étudier le droit international privé en 1897 (Yamada 1929 : 76), il participe à la conférence de Bruxelles de 1897 sur la propriété industrielle en compagnie de Motono Ichirō 本野一郎 (1862$1918)^{21}$, ministre plénipotentiaire à Bruxelles, et de "Soughi Takejiro ${ }^{22}$ " (sic), examinateur du Bureau des brevets d'inventions au ministère de l'Agriculture et du Commerce (Union internationale pour la Protection de la propriété industrielle 1901 : 365). Par la suite, il séjourne pendant plus d'une année aux alentours de 1900 à Paris et suit les cours de la faculté de droit (Yamada 1929 : 17). À cette occasion, il participe au Congrès de la propriété littéraire et artistique de Paris qui se tient du 6 au 21 juillet 1900 (Bulletin de l'Association littéraire et artistique 1900 : 19). Secrétaire, il y présente la législation japonaise du droit d'auteur lors de la séance du 20 juillet (Yamada 1900 : 60). Quelques jours plus tard, il est l'un des vice-présidents d'honneur du Congrès international de la propriété industrielle qui se tient à Paris du 23 au 28 juillet 1900, où il est présenté comme " professeur de droit national $(s i c)$ privé à l'université impériale de Tokyo et conseiller en droit au Bureau des brevets d'invention au ministère de l'Agriculture et du Commerce du Japon » (Le Soudier 1901 : 13). Il est ainsi présent aux premières conventions internationales en matière de propriété intellectuelle, en tant que représentant du Japon. Celui-ci s'avère être le premier pays d'Asie - avec cent ans d'avance - à accorder autant d'importance aux droits de propriété intellectuelle.

Yamada Saburō est nommé à la tête de la "Commission nationale de coopération internationale »(La Presse, 16 juillet 1926), émanation japonaise de la Commission internationale de coopération intellectuelle

21. Licencié de la faculté de droit de Lyon, il y avait accompli ses études avec Ume Kenjirō et entrera dans la diplomatie.

22. Sugi Takejirō 杉竹二郎 [N.D.L.R.]. 
(CICI), créée en janvier 1922, qui deviendra l'Unesco en 1946. En 1939, il est proposé comme recteur de l'université de Tokyo par ses collègues, après avoir été recteur de l'université de Keijō (Séoul) d'octobre 1931 à janvier 1936, mais le général Araki, ultra-nationaliste et alors ministre de l'Éducation, ne donna pas son approbation (Bulletin périodique de la presse japonaise 1939/03/09-1939/03/11 : 17). Après la guerre, il ne cesse d'occuper de très importantes fonctions. Il est notamment nommé président de l'Académie japonaise des sciences en juin 1948 et le restera jusqu'en novembre $1961^{23}$. Il est par ailleurs fait docteur honoris causa de l'université de Paris en 1959. Lorsque, en fin de carrière, il redevint très actif au sein de la Maison, il est spécialement proche de Frédéric Joüon de Longrais, des «liens fondés autant sur l'estime et la sympathie personnelle que sur une même ferveur pour l'étude, et qu'une fidélité réciproque dans l'épreuve élèvera plus tard au niveau supérieur d'une sorte de fraternité » (Frank et Boussard 1976 : 50). Il est nommé cinquième président du Conseil d'administration de la Maison en 1946, ne quittant cette fonction qu'en 1962 (Iyanaga : 15). Malheureusement sa gestion sera très discrète et il ne laisse rien en français sur sa direction et sa présence à la MFJ.

Nous pouvons donc dire que si leur présence est de nos jours plus discrète, les juristes ont ainsi porté la Maison franco-japonaise sur les fonts baptismaux, puis l'on nourrie.

\section{Conclusion}

S'arrêter un instant sur la présence et le rôle des juristes au sein de la Maison franco-japonaise permet de mettre en lumière certains de ses aspects : la diffuse présence de Lyon dans les statuts et l'histoire de l'Institution, le fait qu'elle ait autant attiré les juristes français alors que ceux-ci sont peu enclins aux échanges internationaux et, enfin, le déséquilibre dans la structure juridique de l'établissement entre la partie française et la partie japonaise. La présence des juristes au sein de la MFJ s'est maintenue et continue, bien que les juristes français y soient moins représentés ces dernières décennies; en

23. Hozumi l'avait été d'octobre 1917 à octobre 1925 ; cf. le site de l'Académie japonaise : http://www.japan-acad.go.jp/en/about/successive.html 
revanche, la présence de la ville de Lyon s'est éclipsée au fil des années, au profit de la capitale.

À travers la fondation de la Maison franco-japonaise, les intellectuels et les mécènes japonais souhaitent rendre un hommage à leurs professeurs français, certes, mais une autre hypothèse nous semble émerger : les initiateurs japonais souhaitaient parallèlement susciter la reconnaissance qui leur était due. De même qu'une motivation essentielle des Français dans leur soutien à ce projet était la recherche d'un rayonnement académique international.

Inviter des universitaires français de renom est aussi le moyen de faire connaître en France - et donc en Europe - la formidable évolution que connaît le Japon. En 1922, lors du cinquantenaire de la Société de législation comparée, Sugiyama déclare :

« la rapidité des progrès de notre civilisation surpassant tous les exemples analogues dans l'histoire du monde, on est tenté, ou bien de se demander si ce progrès n'est pas un phénomène tout à fait superficiel, ou bien de renoncer à en chercher la cause comme s'il y avait là une énigme insoluble; ou bien encore de craindre que la civilisation du nouveau Japon ne disparaisse subitement, telle une comète qui n’a fait que briller quelque temps » (Sugiyama $1922: 221$ )

Il affirme ensuite vouloir " préciser les causes et les résultats de ce développement inouï du droit japonais» (Sugiyama 1922 : 222).

Plus de trente ans après le départ de Boissonade, les sciences juridiques se sont considérablement développées, elles sont enseignées depuis plusieurs décennies dans des facultés qui se sont multipliées au Japon. Les juristes occupent une place très importante dans le monde politique car ce sont les facultés de droit qui forment les diplomates et les hauts fonctionnaires c'est probablement l'une des raisons pour lesquelles plusieurs d'entre eux soutiendront ardemment le projet. L'on peut penser qu'après plus de cinquante ans de relations, les juristes japonais, à travers la Maison, cherchent à installer une relation enfin devenue réciproque et fondée sur un principe d'égalité avec leurs pairs français. 


\section{Bibliographie}

\section{Archives}

Archives de la Maison franco-japonaise, Bureau français

Archives départementales du Rhône Fonds Professeur Louis Josserand, doyen de la faculté de droit de Lyon (1897-1941), $224 \mathrm{~J} 1-67$; répertoire numérique détaillé établi par Éric Montat, édition de documents choisis réalisée par Catherine Fillon, 2012 : http://cg69-jpg2.naoned-systemes.fr/ accounts/mnesys_cg69/datas/medias/IR_ pour_internet/224\%20J.pdf

Archives du musée Guimet

Archives personnelles René Garraud

\section{JACAR}

Japan Center for Asian Historical Records, National Archives Japan 国立公文書館 アジア歴史資料センター

Papiers Kikou Yamata $\left(19^{\mathrm{e}}-20^{\mathrm{e}}\right)$ Fonds CH BGE Ms. fr. 6321-6355, Bibliothèque de Genève, http://w3public.ville-ge.ch/bge/odyssee. nsf/Attachments/yamata_kikouframeset. htm/\$file/yamata_kikou.htm
Shibusawa Eiichi denki shiryō 渋沢栄一伝記資料

(Documents biographiques Shibusawa Eiichi)

Dai 36 kan dai 11 kan Nichifusu kyōkai 第36巻 第11款 日仏協会 (vol. 36, chap. 11 : La Société franco-japonaise) ; Dai 36 kan dai 12 kan Zaidan hōjin Nichifutsu kaikan 第36巻 第12款 財団法人 日仏会館 (vol. 36, chap. 12 : La Fondation (Société) Maison franco-japonaise).

\section{Presse \& Divers}

Annuaire de l'université de Lyon, 1934 Livret de l'étudiant, publié par les bons soins du Conseil général des facultés, année scolaire 1934-1935.

\section{Annuaire Saint-Étienne, 1908}

Annuaire administratif, commercial, industriel et statistique du département de la Loire et Indicateur de Saint-Étienne réunis.

Bulletin de l'Association littéraire et artistique internationale, 1900

Bulletin périodique de la presse japonaise, 1939

Ministère des Affaires étrangères, 1939/03/09-1939/03/11 


\section{Ouvrages et articles}

\section{ALLIX Edgar, 1934}

«Allocution à l'occasion de la réception du Professeur Sugiyama », Chronique de l'Université, Annales de l'université de Paris 1934, Paris, Société des amis de I'Université, Bureau des renseignements scientifiques de l'université de Paris, pp. 185-190.

\section{AUDREN Frédéric}

\& HALPÉRIN Jean-Louis, 2013

La culture juridique française. Entre mythes et réalités, $X I x^{e}$ et $x X^{e}$ siècles, Paris, CNRS éditions.

\section{BERTHÉLÉMY Henri, 1932}

"Discours à M. Sugiyama ", Annales de I'université de Paris, pp. 494-497.

\section{BOUCHEZ Daniel, 1984}

« Un rapport de Maurice Courant sur la mission de $1919 »$, Nichifutsu bunka, 45 : 31-58.

CAPITANT Henri アンリ•カピタン, 1931 "Kindai ni okeru Furansu minpō no hensen » 近代に於けるフランス民法の 変遷 («Les transformations du droit civil français depuis cinquante ans »), traduction japonaise par Sugiyama Naojirō 杉山直治郎, Nichifutsu bunka 日仏文化, Shin dai isshū 新第1輯 ( $n^{\circ} 1$ Nouvelle Série) : 17-81.

\section{CHABAS François, 1933}

«De la responsabilité civile et délictuelle en droit comparé francais-japonais ", Bulletin de la Maison franco-japonaise, Série française, 5 (3).
CHŪJŌ Shinobu, 2001

«Paul Claudel et la fondation de la Maison franco-japonaise », Ebisu. Études japonaises, 26 : 7-34.

CHŪJŌ Shinobu, HOURIEZ Jacques \& BAZAUD Maryse, 2003

«Correspondance Léonard-Eugène Aurousseau - Paul Claudel (19221926) », Ebisu. Études japonaises, 30 : 179-215.

\section{CFEUR Pierre, 2005}

Mémento de l'histoire de Lyon, Brignais, éd. Des Traboules.

\section{COURANT Maurice, 1919}

"La mission universitaire lyonnaise au Japon (1919) », rapport inédit publié dans BOUCHEZ Daniel, 1984, « Un rapport de Maurice Courant sur la mission de 1919 », Nichifutsu bunka, $45: 31-58$.

\section{DUROZOI Gérard} \& BOUVET Vincent, 2009 Paris, 1919-1939, Paris, éd. Hazan.

\section{FILLON Catherine, 2014}

"Les prodromes de la Maison francojaponaise de Tokyo : I'université de Lyon et le Japon à la fin de la Première Guerre mondiale », in B. JALUZOT (dir.), Droit japonais, droit français, quel dialogue?, Zurich, Ed. Schulthess, pp. 37-54.

FRANK Bernard, 1974

«La Maison franco-japonaise, son histoire, ses buts, son fonctionnement ", Nichifutsu bunka, 31 : 1-103. 
FRANK Bernard

\& BOUSSARD Jacques, 1976

« Joüon des Longrais Frédéric (24

avril 1892-13 février 1975) », Annuaire

1975-1976. École pratique des hautes

études Ive section. Sciences historiques et

philologiques, p. 50 et s.

\section{HARA Terushi, 1996}

«Les facteurs psychologiques et culturels de la modernisation du Japon : le cas de Eiichi Shibusawa », in R. BOUDON \& P. CHAUNU (dir.), Autour de Alain Peyrefitte. Valeurs et modernité, Paris, Odile Jacob, pp. 113-132.

IYANAGA Sokichi, 1984

«L'évolution de la Maison francojaponaise », Nichifutsu bunka, 45 : 15-26.

\section{JALUZOT Béatrice, 2014}

«Tomii et Ume : quand la faculté de droit de Lyon forme les rédacteurs du Code civil japonais », in B. JALUZOT (dir.), Droit japonais, droit français, quel dialogue?, Zurich, Ed. Schulthess, pp. 5-19.

\section{JOUBIN Paul, 1929}

"Correspondance ", La Revue de Paris, p. 948 et s.

\section{JOÜON DES LONGRAIS Frédéric, 1947}

« L'œuvre scientifique de la Maison franco-japonaise 1939-1947 », Compterendu des séances de l'académie des Inscriptions et des Belles Lettres, 91 (2) : 294-301.

\section{JULLIOT DE LA MORANDIÈRE Léon, 1939}

« Hommage au Professeur Henri

Capitant », in JULLIOT DE LA

MORANDIÈRE Léon \& RAY Jean (dir.),

Hommage au baron Tomii et au

professeur Capitant, Société franco-

japonaise, Institut d'études japonaises

de l'université de Paris, p. 21 et s.

\section{JULLIOT DE LA MORANDIÈRE Léon, 1966}

"Nécrologie : Naojiro Sugiyama », Revue internationale de droit comparé, 18 (4) : 923-927.

\section{LE SOUDIER Henri, 1901}

Congrès international de la propriété industrielle, organisé par la commission permanente internationale de la propriété industrielle avec le concours de l'Association internationale pour la protection de la propriété industrielle et de l'Union des fabricants, à Paris, du 23 au 28 juillet 1900, monographie imprimée, Paris.

\section{OKUBO Yasuo, 1991}

« La querelle sur le premier Code civil japonais et l'ajournement de sa mise en vigueur: le refus du législateur japonais ? ", Revue internationale de droit comparé, 43 (2) : 389-405.

\section{LÉVI Sylvain, 1929}

"La Maison franco-japonaise de Tokyo ", Revue de Paris, 15 sept. 1929 : 410 et s.

MALHERBE Marc, 1996

La Faculté de droit de Bordeaux, 1870-1970, Bordeaux, Presses universitaires de Bordeaux. 
MARNOT Bruno, 2012

La mondialisation au XIX ${ }^{e}$ siècle, 1850-

1914, Paris, éd. A. Colin.

MONESTIER Alphonse, 1921

La mission Painlevé en Chine, Pékin, coll. « Politique de Pékin ».

\section{OMOTO Keiko}

\section{\& MACOUIN Francis, 2001}

Quand le Japon s'ouvrit au monde.

Émile Guimet et les arts de l'Asie, Paris,

Gallimard, coll. Découvertes Gallimard, $2^{\mathrm{e} e ́ d .}$

\section{OSMAN Filali, 2014}

«La contribution de Masaichirō Ishizaki

à la doctrine de la lex mercatoria », in

B. JALUZOT (dir.), Droit japonais, droit français, quel dialogue?, Zurich, Ed.

Schulthess, 2014, pp. 79-94.

PELLETIER André, ROSSIAUD Jacques, BAYARD Françoise, CAYEZ Pierre, 2007 Histoire de Lyon des origines à nos jours, Lyon, Ed. Lyonnaises d'Art et d'Histoire.

\section{RAY Jean, 1939}

"Hommage au baron Tomii », in

Hommage au baron Tomii et au

professeur Capitant, Société franco-

japonaise, Institut d'études japonaises

de l'université de Paris, p. 9 et s.

\section{REYNS-CHIKUMA Chris, 2005}

Images du Japon en France et ailleurs.

Entre japonisme et multiculturalisme,

Paris, L'Harmattan.

SUGIYAMA Naojirō, 1922

« L'évolution générale du droit japonais moderne (1869-1919) ", in Cinquantenaire de la Société de législation comparée, Paris,

LGDJ, t. II, pp. 219-252.

\section{SUGIYAMA Naojirō, 1936a}

«Les relations intellectuelles entre le Japon et la France : leur origine, leur état actuel et leurs perspectives d'avenir », in Ma mission en France 1934. Conférences et allocutions, Tokyo, Maison franco-japonaise, pp. 101-162.

\section{SUGIYAMA Naojirō, 1936b}

"Les transformations du droit civil français et l'influence du droit français ", Annales de l'Institut de Droit comparé de Paris, p. 195 et s.

\section{SUGIYAMA Naojirō (dir.)}

杉山直治郎 (編), 1936c

Tomii danshaku tsuitōshū 富井男爵追悼集 (Études en mémoire du baron Tomii), Tokyo, Nichifutsu kaikan 日仏会館, Yūhikaku 有斐閣.

\section{SUGIYAMA Naojirō, JULLIOT DE LA MORANDIÈRE Léon, 1936}

"Le baron Tomii », in Le baron Masaakira Tomii (1858-1935), Bulletin de la Maison franco-japonaise, Tokyo, VII (3-4) : 9 et s.

\section{TANAKA Sadao,}

«Émile-Louis Heck : premier professeur de littérature française à l'université impériale de Tokyo et grand directeur de l'Étoile du Matin », en ligne : http://libir.soka.ac.jp/dspace/ bitstream/10911/1075/1/KJ00005448759. pdf 
TOMII Masaakira, 1887

Le Shintoïsme, sa mythologie et sa

morale, Annales du musée Guimet, Paris,

E. Leroux éditeur.

Union internationale

pour la protection de la propriété

industrielle, 1901

Actes de la conférence réunie à Bruxelles

du $1^{\text {er }}$ au 14 décembre 1897 et du 11

au 14 décembre 1900, Berne, Bureau

international de l'Union.

VINCENT Jean-François, 1995

La bibliothèque de la Maison franco-

japonaise, Mémoire d'étude, ENSSIB.

\section{YAMADA Saburō, 1900}

"La propriété littéraire et artistique au Japon ", Bulletin de l'Association

littéraire et artistique internationale, pp. 60-64

\section{YAMADA Saburō, 1929}

"La Maison franco-japonaise", conférence prononcée à la faculté de droit de Paris le 6 mai 1929, Bulletin de la société franco-japonaise de Paris, p. 17 et s. 